\title{
Successful treatment of pulmonary zygomycosis in two transplant recipients with liposomal amphotericin B and partial surgical resection followed by posaconazole
}

\author{
David T. Cooke, ${ }^{1}$ Francis D. Pagani, ${ }^{2}$ Daniel R. Kaul, ${ }^{3}$ Kenneth R. Cooke, ${ }^{4}$ Christine L. Lau ${ }^{5}$ and James \\ Riddell IV ${ }^{3}$ \\ ${ }^{1}$ Division of Cardiothoracic Surgery, University of California, Davis Medical Center, Davis, CA, USA, ${ }^{2}$ Section of Cardiac Surgery, University of Michigan Medical \\ Center, Ann Arbor, MI, USA, ${ }^{3}$ Division of Infectious Diseases, University of Michigan Medical Center, Ann Arbor, MI, USA, ${ }^{4}$ Department of Pediatrics, Case \\ Western Reserve University School of Medicine, Cleveland, OH, USA and ${ }^{5}$ Division of Thoracic and Cardiovascular Surgery, University of Virginia, Charlotte, VA, \\ USA
}

\section{Summary}

Pulmonary zygomycosis is a relatively uncommon complication of solid organ or peripheral blood stem cell transplantation and has a high associated mortality. Optimal therapy consists of complete resection of infected tissue and treatment with amphotericin B (AmB). We describe two patients, one of whom underwent orthotopic heart transplantation and the other who received a peripheral blood stem cell transplant, who were diagnosed with invasive pulmonary zygomycosis. Both patients were treated with a liposomal preparation of $\mathrm{AmB}$ and early partial resection of the infected structures followed by prolonged posaconazole maintenance therapy. Despite incomplete resection, this treatment regimen resulted in a favourable outcome in both patients, including survival of more than 17 months in one patient at last follow up. For patients in whom complete resection of pulmonary zygomycosis is not possible, subtotal resection and treatment with liposomal AmB followed by therapy with posaconazole may be an effective treatment option.

\section{Introduction}

Pulmonary zygomycosis is a rare complication of transplantation with an associated mortality of up to $50 \%$ when treated with amphotericin B (AmB) alone (Almyroudis N, Sutton D, Linden P, Rinaldi M, Fung J, Kusne S. Am J Transplant 2006; 6: 2365-74). Complete excision of infected tissue, combined with the administration of liposomal AmB has been shown to reduce mortality to $6 \%$ in patients with isolated pulmonary infection (Almyroudis N, Sutton D, Linden P, Rinaldi M, Fung J, Kusne S. Am J Transplant 2006; 6: 2365-74). The role of surgical debridement for patients with

Correspondence: Christine Lau, Division of Thoracic and Cardiovascular Surgery, University of Virginia, PO Box 800679, Charlottesville, VA 229080679, USA.

Tel.: +1 4139248016 . Fax: +1 4342436131.

E-mail: cll2y@virginia.edu

Accepted for publication 4 November 2008 unresectable disease remains unclear. The role of the new triazole agent, posaconazole for the treatment of infections with zygomycetes is also yet to be defined. In this report, we present two transplant recipients (one solid organ transplant and one allogeneic stem cell transplant) with invasive pulmonary zygomycosis. Because of the disease extent and anatomical structures involved in the infection, both patients could only undergo subtotal resection of infected tissue. Each patient was treated with liposomal amphotericin B (Ambisome) followed by a prolonged course of posaconazole and had a favourable response to therapy.

\section{Case reports}

\section{Case 1}

A 48-year-old man who underwent an orthotopic heart transplant with thymoglobulin induction for ischaemic cardiomyopathy presented 2 months later with right 


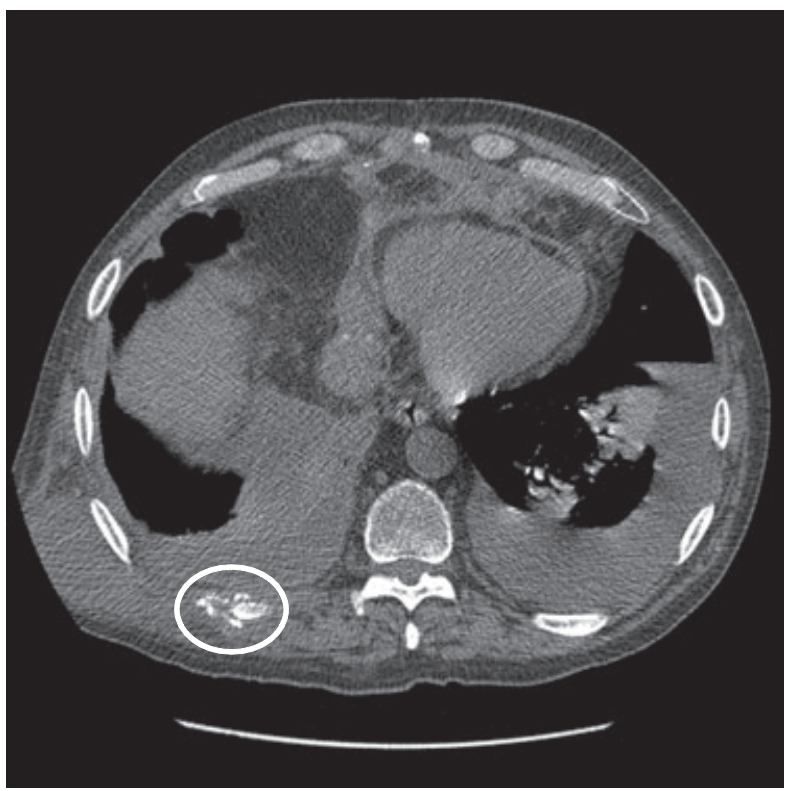

Figure 1 Axial computed tomography (CT) image of the chest demonstrating erosion of the ninth rib (circle) with an associated mass.

posterior chest wall tenderness and a non-productive cough while on maintenance immunosupression with tacrolimus and prednisone $10 \mathrm{mg}$ twice per day. A computed tomography (CT) scan demonstrated multilobar infiltrates, cavitary masses in the right upper lobe and right ninth rib destruction with an associated mass (Fig. 1). Culture of aspirated fluid from a right sided pleural effusion yielded Mucor spp. The patient underwent right lateral chest wall resection. Complete resection could not be achieved because of extensive chest wall involvement and multi-lobar disease with pleural infection. Histology from operative specimens was consistent with invasive zygomycosis. Multiple intraoperative cultures of tissue and fluid yielded Mucor spp. The patient was initially treated with a 12-day course of liposomal AmB (Ambisome) $10 \mathrm{mg} \mathrm{kg}^{-1} \mathrm{day}^{-1}$, which was discontinued because of nephrotoxicity (increase of creatinine from 1.8 to $2.6 \mathrm{mg} \mathrm{dl}^{-1}$ ), and posaconazole $200 \mathrm{mg}$ p.o. four times per day was begun on postoperative day (POD) 11. The patient recovered and was discharged on POD 21. He returned on POD 33 with a recurrent para-pneumonic effusion that again yielded Mucor spp. on fungal culture. An empyema tube was placed and a second course of liposomal AmB $5 \mathrm{mg} \mathrm{kg}^{-1}$ day $^{-1}$ was attempted, again complicated by nephrotoxicity (increase of creatinine to $4.8 \mathrm{mg} \mathrm{dl}^{-1}$ ), and followed by treatment with posaconazole $200 \mathrm{mg}$ p.o. four times per day. The empyema tube was removed 3 months later. A CT scan 4 months after his original surgery revealed evidence of a favourable response to therapy (Fig. 2). The patient remains asymptomatic on posaconazole therapy $400 \mathrm{mg}$ p.o. twice per day with continuing regression of his remaining pulmonary disease almost 2 years after subtotal resection of the infected tissue.

\section{Case 2}

A 32-year-old man, 34 days status postallogeneic bone marrow transplant (BMT) for aplastic anaemia complicated by engraftment syndrome and grade II graftversus-host disease (GVHD) of skin treated with tacrolimus, Enbrel, and medrol with voriconazole antifungal prophylaxis, presented with a persistent cough. Absolute neutrophil count was $2700 \mathrm{~mm}^{-3}$ and the absolute lymphocyte count was $500 \mathrm{~mm}^{-3}$. A chest CT scan revealed a mass surrounding the right lower lobe (RLL) bronchus with an adjacent RLL pulmonary artery aneurysm (Fig. 3). The aneurysm was coil embolised and the next day a RLL and right middle lobe bilobectomy was performed. Pathological examination revealed aseptate hyphae with broad angle branching consistent with zygomycosis with pulmonary artery and bronchial invasion (Fig. 4). Unresectable fungal tissue invaded the
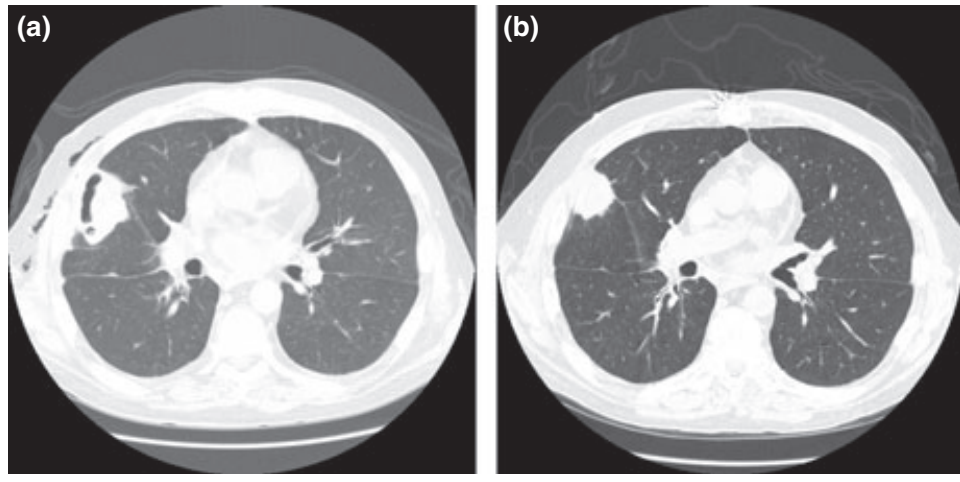

Figure 2 CT image of the chest demonstrating a cavitary parenchymal mass and pleural fluid in the major fissure and pleural space (a). CT image of same anatomic location 4 months after $\mathrm{AmB}$ and posaconazole therapy (b). There is marked reduction of Mucor burden and pleural fluid. 


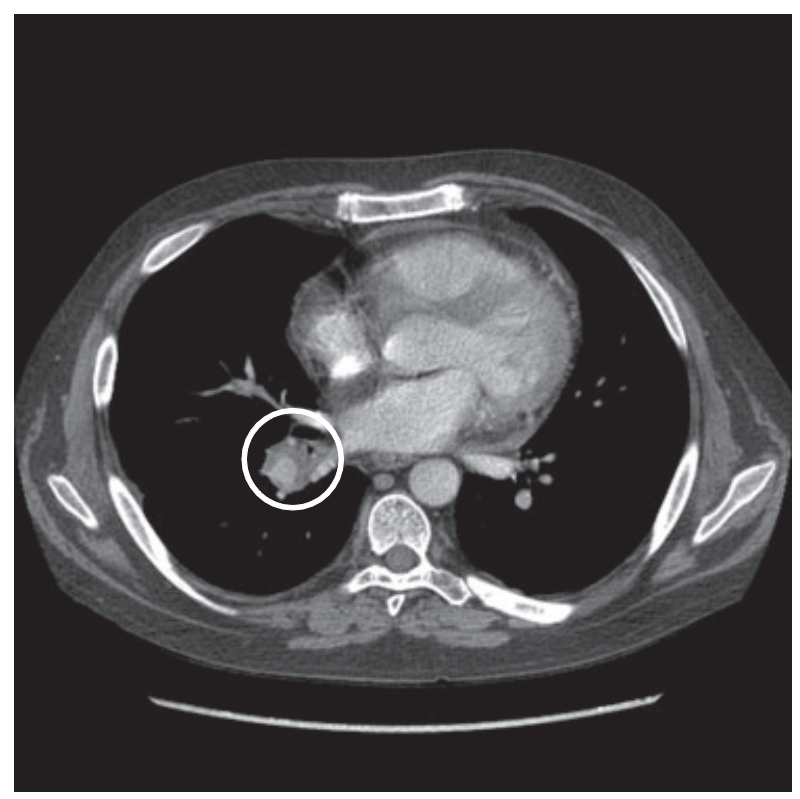

Figure 3 CT image demonstrating a PA aneurysm and hilar mass (circle). PA, pulmonary artery.

hilum, which would have necessitated a pneumonectomy in an infected field. A culture was not performed at the time of the operation. The patient received 4 weeks of therapy with liposomal AmB $10 \mathrm{mg} \mathrm{kg}^{-1} \mathrm{day}^{-1}$, which was discontinued when an adequate radiographic response was documented and it was necessary to initiate treatment with foscarnet. After amphotericin was discontinued, treatment with posaconazole $200 \mathrm{mg}$ p.o. four times per day was initiated. He returned 1 month later with methicillin-resistant Staphylococcus

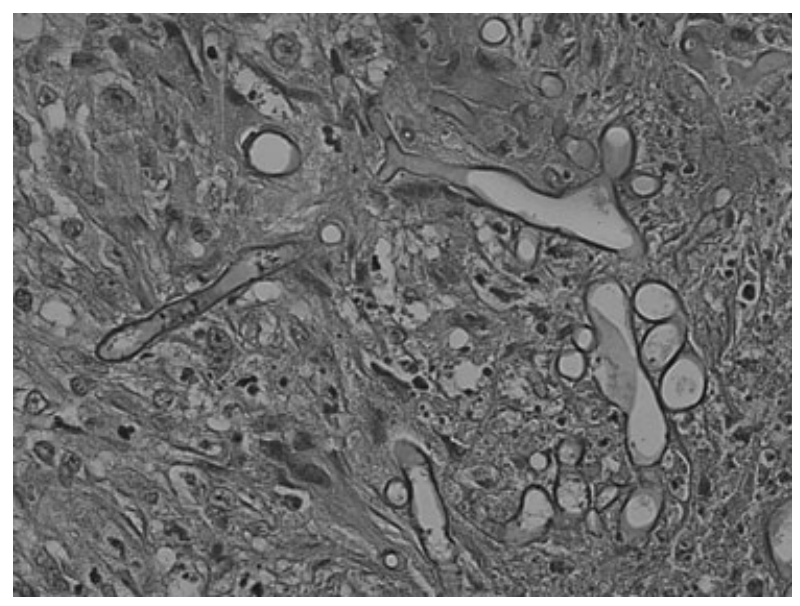

Figure 4 Invasion of hilar tissue with broad aseptate hyphae with $90^{\circ}$ angle branching consistent with a zygomycete $(500 \times$ magnification). aureus bacteraemia. On POD 109, the patient died from sepsis. An autopsy was declined by the family.

\section{Discussion}

These case reports demonstrate a successful outcome to treatment of invasive pulmonary zygomycosis in the setting of immune suppression with the use of partial surgical debridement and therapy with AmB followed by long-term treatment with oral posaconazole.

Although the incidence of invasive infection with zygomycetes is generally reported to be less than $1 \%$ in most transplant centres (Uckay I, Chalandon Y, Sartoretti P et al., Clin Transplant 2007; 21: 577-82), associated mortality remains high and the incidence of such infections is increasing (Marr K, Carter R, Crippa F, Wald A, Corey L. Clin Infect Dis 2002; 34: 909-917). In the largest literature review published to date regarding the epidemiology and outcomes of 929 cases of zygomycosis, $6 \%$ of the cases were associated with solid organ transplantation with a mortality of $48 \%$, and $5 \%$ had undergone bone marrow transplantation with 91\% associated mortality (Roden M, Zaoutis T, Buchanan W et al., Clin Infect Dis 2005; 41: 634-53). Overall, the incidence of infection with non-Aspergillus filamentous fungi seems to be increasing, particularly after the introduction of voriconazole for antifungal prophylaxis, which does not have activity against zygomycetes (Siwek G, Dodgson K, de Magalhaes-Silverman M et al., Clin Infect Dis 2004; 39: 584-7; Imhof A, Balajee A, Fredricks D, Englund J, Marr K. Clin Infect Dis 2004; 39: 743-6). Zygomycetes comprised $5.7 \%$ of invasive fungal infections in organ transplant recipients in one recent multicentre study (Husain S, Alexander B, Munoz P et al., Clin Infect Dis 2003; 37: 221-9). With increasing incidence, as well as an overall high mortality, there is an urgent need to identify more optimal strategies for the management of infection with zygomycetes.

The traditional approach to therapy has consisted of complete surgical resection of infected tissue with the concomitant administration of an AmB product (Tedder M, Spratt J, Anstadt M et al., Ann Thorac Surg 1994; 57: 1044-50). Often, however, invasive fungal infections cross tissue planes and can involve multiple noncontiguous structures, which complicate decisions regarding surgical debridement. In many cases, if complete resection of the involved tissue cannot be accomplished, surgical intervention is deferred. Many studies have demonstrated the benefit of debridement, particularly with pulmonary disease. Mortality was reduced from $50 \%$ with $\mathrm{AmB}$ alone to $6.6 \%$ with 
AmB plus surgery for pulmonary zygomycosis in one retrospective review of solid organ transplant recipients (although patients with fewer comorbidities were more likely to be the candidates for surgery) (Almyroudis N, Sutton D, Linden P, Rinaldi M, Fung J, Kusne S. Am J Transplant 2006; 6: 2365-74). In another small study of solid organ transplant recipients, the two patients with pulmonary zygomycosis who underwent surgical resection survived, while the two who did not expired (Stelzmueller I, Lass-Floerl C, Geltner C et al., Transpl Internat 2008; 21: 534-46). A study in renal transplant recipients, however, did not demonstrate such a beneficial effect. Of four patients with pulmonary disease, only one of the three patients who underwent upper lobectomy or wedge resection survived (Forrest G, Mankes K. Transpl Infect Dis 2007; 9: 161-4). Because invasive zygomycosis is rare, clinical decisions with regards to management are often reliant upon case reports as it is not possible to conduct prospective trials. Our case report combined with the above reported experience suggests that partial resection combined with medical therapy is a reasonable option for patients in whom complete resection is not possible.

Clearly, appropriate antifungal therapy also remains an important component of treatment. Although some species such as Cunninghamella are less responsive to antifungal therapy of any type, an amphotericin product is considered the first line therapy for all zygomycete species (Ribes J, Vanover-Sams C, Baker D. Clin Microbiol Rev 2000; 13: 236-301). A recent study has shown that the timing of the initiation of therapy is also critical to outcomes. In patients with haematological malignancy with zygomycosis, a delay in treatment by greater than 6 days doubled 12-week mortality compared with those who received prompt initiation of therapy (Chamilos G, Lewis R, Kontoyiannis D. Clin Infect Dis 2008; 47: 503-9). In this same study, posaconazole use after initial treatment with amphotericin was associated with a favourable outcome (Chamilos G, Lewis R, Kontoyiannis D. Clin Infect Dis 2008; 47: 503-9). Posaconazole has been shown to have in vitro activity against zygomycetes with $\mathrm{MIC}_{50}$ levels of $1 \mu \mathrm{g} \mathrm{ml}^{-1}$ or less for these organisms (Sabatelli F, Patel R, Mann P et al., Antimicrob Agents Chemother 2006; 50: 2009-15; Dannauoui E, Meletiadis J, Mouton J, Meis J, Verweij P, Network E. J Antimicrob Chemother 2003; 51: 45-52). Although there have been no long-term comparative studies, observational studies have demonstrated clinical responses of invasive zygomycete infections to posaconazole. Of 24 patients with invasive zygomycete infection who participated in the posaconazole expanded access protocol who were failing or intolerant of amphotericin, 17 (71\%) were found to have partial or complete remission of infection (Greenberg R, Anstead G, Herbrecht R et al., Posaconazole (POS) experience in the treatment of zygomycosis. Program and Abstracts of the 43rd Annual ICAAC, Chicago, IL; Abstract M-1757: September 14-7, 2003). Posaconazole has also been shown to have an excellent safety profile in this population (Raad I, Graybill J, Bustamante A et al., Clin Infect Dis 2006; 42: 1726-34). The two patients included in this report add to the accumulated data that posaconazole is a useful adjunctive therapy for infection with zygomycetes.

Because infection with filamentous fungi in high-risk populations carries such a high degree of morbidity and mortality, novel approaches to treatment and prophylaxis have been investigated. The second patient developed invasive infection with a zygomycete while on voriconazole prophylaxis, which does not have activity against these organisms. The use of posaconazole for prophylaxis may be more effective at preventing such infections in stem cell recipients with GVHD (Ullmann A, Lipton J, Vesole D et al., New Engl J Med 2007; 356: 335-47). With regards to therapy, a degree of improved efficacy has been observed with the use of combination therapy with two antifungal agents (voriconazole plus an echinocandin) for invasive aspergillosis in patients who failed initial treatment with AmB (Marr K, Boeckh M, Carter R, Kim H, Corey L. Clin Infect Dis 2004; 39 : 797-802). Even though the echinocandins do not demonstrate in vitro activity against zygomycetes (Espinel-Ingroff A. J Clin Microbiol 1998; 36: 2950-6), the target enzyme of echinocandins has been shown to be present in this species and a clinical response has been demonstrated in murine models (Ibrahim A, Bowman J, Avanessian V et al., Antimicrob Agents Chemother 2005; 49: 721-7). In the first clinical study of its kind, patients with rhino-orbital-cerebral mucormycosis who received both AmB and caspofungin were analysed in a retrospective fashion compared with those who received AmB alone. Of 41 patients in total, all six who received combination therapy survived vs. $45 \%$ of those who received only an AmB preparation (Reed C, Bryant R, Ibrahim A et al., Clin Infect Dis 2008; 47: 364-71). The combination of posaconazole and amphotericin has not been evaluated in clinical studies. Because of concerns for potential antagonism, this combination is not commonly used in practice.

It would therefore appear that optimal therapy for pulmonary invasive zygomycete infection in the setting of significant immune compromise such as solid organ transplantation or bone marrow transplant would 
involve a combination of the prompt initiation of an AmB preparation followed by complete or partial surgical debridement. A course of therapy with AmB could be followed by treatment with posaconazole until complete clinical resolution of the infection is documented radiographically. As most transplant patients require long-term immune suppression, the ultimate duration of oral therapy remains unclear because relapse of fungal infections can occur. The role of combination antifungal therapy has not yet been clarified; however, this may be an attractive option for refractory cases.

\section{Acknowledgment}

We would like to acknowledge Carrie Lapham for her assistance in preparing this manuscript. 